\title{
Samuel Beckett and Contemporary Art, Robert REGINIO, David Houston JonEs, Katherine Weiss
}

\section{Stefano Genetti}

\section{CpenEdition \\ Journals}

\author{
Edizione digitale \\ DOI: 10.4000/studifrancesi. 15811 \\ ISSN: 2421-5856 \\ Editore \\ Rosenberg \& Sellier

\section{Edizione cartacea} \\ Data di pubblicazione: 1 décembre 2018 \\ Paginazione: $528-529$ \\ ISSN: 0039-2944
}

URL: http://journals.openedition.org/studifrancesi/15811

\section{Notizia bibliografica digitale}

Stefano Genetti, «Samuel Beckett and Contemporary Art, Robert Reginıo, David Houston Jones, Katherine WEISS», Studi Francesi [Online], 186 (LXII | III) | 2018, online dal 01 janvier 2019, consultato il 06 janvier 2021. URL: http://journals.openedition.org/studifrancesi/15811 ; DOI: https://doi.org/10.4000/ studifrancesi. 15811

Questo documento è stato generato automaticamente il 6 janvier 2021.

\section{(c) (i) (9)}

Studi Francesi è distribuita con Licenza Creative Commons Attribuzione - Non commerciale - Non opere derivate 4.0 Internazionale. 


\title{
Samuel Beckett and Contemporary Art, Robert REGINIO, David HousTON JONES, Katherine WEISS
}

\author{
Stefano Genetti
}

\section{NOTIZIA}

Samuel Beckett and Contemporary Art, Robert REGINIO, David Houston JonES, Katherine WEIss, Stuttgart (eds), ibidem-Verlag, 2017, 367 pp.

1 Una scatola nera, scarsamente illuminata, dove tableaux vivants si animano e si dissolvono, dove l'estrema economia degli elementi drammaturgici, la loro ripetizione e permutazione, nonché la tensione tra tridimensionalità del palcoscenico e bidimensionalità dell'immagine, suscitano un'interrogazione innanzi tutto percettiva dell'evento visivo: è in un'ottica spaziale che il ruolo svolto da Beckett nell'ambito dell'arte minimalista e concettuale si impone, in riferimento alle sue creazioni per la scena e per lo schermo dagli anni Sessanta in poi e per analogia con le modalità espositive della video-installazione. È quanto sottolinea, in margine all'annoso dibattito su Modernismo e Postmodernismo, Robert REGINIO nella sua Introduction (pp. 9-33) a questa stimolante e coesa raccolta di saggi, dove ritornano frequentemente le questioni della figuratività e dell'astrazione, dell'autoriflessività e della serialità, delle dialettiche trasparenza/opacità e persistenza/debilitazione del visibile, della multisensorialità e della transmedialità, in relazione a nomi quali Sol LeWitt (R. REGINIO, Specific Objects and Inscrutable Activities: Beckett in the Landscape of Post-War American Art, pp. 107-132; si veda, a p. 130, il disegno per il dramma Come and Go/Va-et-vient), Donald Judd (Zachary TAVlin, Kirin WACHTER-GReNe, Theatrical Minimalisms: On Place in Beckett and Judd, pp. 87-106), Doris Salcedo e Mona Hatoum (David Houston Jones, A Singular Totality: Beckett, Salcedo, Hatoum and Installation Art, from Closed System to Embodied Cognition, pp. 135-153), ma anche in relazione agli artisti le cui dichiarazioni e testimonianze sono 
riunite nella terza e ultima sezione «Artists on Beckett». Si tratta di Paul Chan che, nel novembre del 2007, a stretto contatto con la comunità locale, ha allestito Godot nella New Orleans devastata dall'uragano Katrina (pp. 293-314); del videoartista canadese Stan Douglas, precoce curatore, nel 1988, della mostra Samuel Beckett: Teleplays (pp. 315-333); di Arnold Dreyblatt, artista-compositore attivo a Berlino che riconfigura le geometrie della prosa descrittiva Imagination Dead Imagine (Imagination morte imaginez, del 1965, in Têtes-mortes) utilizzando la tecnica del cut-up (pp.335-342); delle irlandesi Dorothy Cross (pp. 343-349) e Mary McIntyre (pp. 351-360) che, tramite mezzi diversi fotografia, video, installazione - si rivelano sensibili alle tracce dei traumi della storia e della violenza politica disseminate nell'opera di Beckett.

2 Nella prime due parti, intitolate rispettivamente «Beckett as Contemporary Artist» e «Beckett in Contemporary Art», della miscellanea-opportunamente illustrata ma sprovvista di indice dei nomi e dove ciascun contributo è accompagnato dalla relativa bibliografia - l'effetto Beckett sul panorama artistico degli ultimi decenni è misurato dapprima in termini di ricezione coeva, di prossimità e di assimilazione-ricreazione delle sue opere, poi in termini di influsso meno diretto e immediato, di ispirazione a distanza e di trasposizione. Si procede così dalla giustapposizione, alle origini del Concettualismo, di The Death of the Author ( $\mathrm{La}$ mort de l'auteur) di Barthes $\mathrm{e}$ dell'interpretazione vocale dell'ottavo dei Textes pour rien di Beckett ad opera dell'attore irlandese Jack MacGowran nella rivista-assemblaggio «Aspen», pubblicazione in scatola che costituiva una sorta di alternativo spazio espositivo in miniatura (Sarah GARLAND, Beckett in a Box: "Texts for Nothing" 8 in Brian O'Doherty's "Aspen" 5+6, pp. 37-63) verso le variazioni sonore elaborate a partire da testi di Beckett e sulle orme dei suoi drammi radiofonici (Derval TURBIDY, Beckett and Sonic Art, pp. 265-290); dagli echi del romanzo Molloy nell'opera di Bruce Nauman, Robert Morris e Claes Oldenburg (Carla TABAN, «Play it again, Sam»: Undoing and Redoing Samuel Beckett's "Molloy" in Contemporary Art, pp.65-85) al riflettersi dell'Occhio-quello di Buster Keaton e quello della cinepresa-protagonista del Film beckettiano nell'occhio di Charlotte Rampling filmato da Steve McQueen (Rachel WelLS, Samuel Beckett and Steve McQueen: Imprisonment, Sight, and Screen, pp. 241-264). Alla nozione di informe derivata da Bataille rinvia Russell SMITH nel commentare alcune operazioni ispirate alla sequenza narrativo-gestuale tipicamente beckettiana camminare-inciampare-cadere-sdraiarsi (manca rimettersi in piedi: Walking...Stumbling...Falling...Lying Down: Beckettian Operations in the Art of John Barbour and Ugo Rondinone, pp. 183-211), mentre Katherine WeIsS si sofferma sull'attività della pittrice, scenografa e costumista svizzera che ha contribuito alla versione televisiva dell'opera di Morton Feldman Neither, su libretto di Beckett (Hessischer Rundfunk, 1990: "gently light unfading»: Traces of Samuel Beckett in the Works of Claire-Lise Hoy, pp. 213-239). Gli sconfinamenti interdisciplinari, le sperimentazioni e le ibridazioni culminano nel saggio che Katharina KNüPPEL dedica, da un lato, al montaggio radiofonico Foursome di Christof Migone, il quale ha invitato quattro coreografi a tradurre verbalmente l'ars combinatoria strettamente cinetica ideata ed esaurita da Beckett nella sua pièce televisiva Quadrat I+II, per quattro interpreti e senza parole; dall'altro, alla collaborazione del video-scultore Peter Welz con il danzatore e coreografo William Forsythe a partire da una frase (whenever on on on nohow on) tratta dal testo in prosa Worstward Ho (Cap au pire). Come osserva R. Reginio (p.128), se è soprattutto in quanto drammaturgo che Beckett partecipa agli sviluppi delle arti visive del secondo Novecento, le sue prose esercitano un influsso durevole su molti artisti 
contemporanei, in questo caso tramite l'interazione di performance danzata e videoinstallazione. 